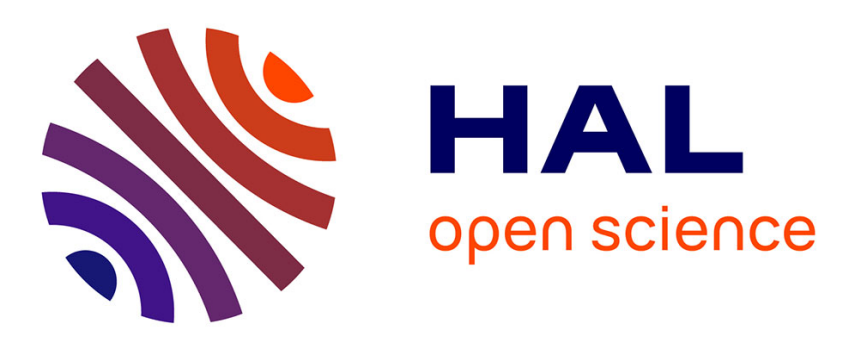

\title{
An incremental-iterative method for modeling damage evolution in voxel-based microstructure models
}

\author{
Q.-Z. Zhu, Julien Yvonnet
}

\section{To cite this version:}

Q.-Z. Zhu, Julien Yvonnet. An incremental-iterative method for modeling damage evolution in voxelbased microstructure models . Computational Mechanics, 2015, 55 (2), pp.371-382. 10.1007/s00466014-1106-1 . hal-01111230

\author{
HAL Id: hal-01111230 \\ https://hal.science/hal-01111230
}

Submitted on 24 Feb 2015

HAL is a multi-disciplinary open access archive for the deposit and dissemination of scientific research documents, whether they are published or not. The documents may come from teaching and research institutions in France or abroad, or from public or private research centers.
L'archive ouverte pluridisciplinaire HAL, est destinée au dépôt et à la diffusion de documents scientifiques de niveau recherche, publiés ou non, émanant des établissements d'enseignement et de recherche français ou étrangers, des laboratoires publics ou privés. 


\title{
An incremental-iterative method for modeling damage evolution in voxel-based microstructure models
}

\author{
Qi-zhi Zhu ${ }^{\mathrm{a}, \mathrm{b}, *}$, Julien Yvonnet ${ }^{\mathrm{b}}$ \\ ${ }^{a}$ College of Civil and Transportation Engineering, Hohai University, 1, Xikang road, 210098 Nanjing, China \\ ${ }^{b}$ Université Paris-Est, Laboratoire Modélisation et Simulation Multi Echelle (MSME), UMR8208 CNRS, 5 Bd Descartes, 77454 Marne-la-Vallée \\ Cedex 2, France
}

\begin{abstract}
Numerical methods motivated by rapid advances in image processing techniques have been intensively developed during recent years and increasingly applied to simulate heterogeneous materials with complex microstructure. The present work aims at elaborating an incremental- iterative numerical method for voxel-based modeling of damage evolution in quasi-brittle microstructures. The iterative scheme based on the Lippmann-Schwinger equation in the real space domain [J. Yvonnet. A fast method for solving microstructural problems defined by digital images: a space Lippmann-Schwinger scheme. Int. J. Numer. Meth. Engng 2012, 92: 178-205.] is first cast into an incremental form so as to implement nonlinear material models efficiently. In the proposed scheme, local strain increments at material grid points are computed iteratively by a mapping operation through a transformation array, while local stresses are determined using a constitutive model that accounts for material degradation by damage. For validation, benchmark studies and numerical simulations using microtomographic data of concrete are performed. For each test, numerical predictions by the incremental-iterative scheme and the finite element method, respectively, are presented and compared for both global responses and local damage distributions. It is emphasized that the proposed incrementaliterative formulation can be straightforwardly applied in the framework of other Lippmann-Schwinger equation-based schemes, like the Fast Fourier Transform (FFT) method.
\end{abstract}

Keywords:

Incremental-iterative scheme, Lippmann-Schwinger equation, Tensile damage, Heterogeneous materials, Tomographic microstructures

\section{Introduction}

In mechanics and material science, recent advances in image processing techniques have permitted direct use of high resolution tomographic images as input data of material internal microstructures for various purposes [1-7]. We mention in particular the voxel-based finite element methods (FEMs) initially motivated in biomechanics [8,9] and

\footnotetext{
${ }^{*}$ Corresponding author

Email addresses: qizhi.zhu@gmail.com (Qi-zhi Zhu), julien.yvonnet@u-pem.com (Julien Yvonnet)
} 
then applied to homogenization of composite materials [10-12] as well as identification of heterogeneous media like concrete $[13,14]$. The basic idea behind these methods is to convert image processing results into a geometric model such that each voxel is used as an element. In other words, the mesh for finite element analyses arises directly from imaging results $[15,16]$. However, structure data yielded from microtomographic techniques usually contain a very large number of material grids points, up to $1024^{3}$ or $2048^{3}$. Accordingly, the global stiffness matrix generated for finite element analyses is usually too large in dimension to be decomposed efficiently for fine models. In addition, the complexity increases when material phases exhibit nonlinear dissipative behaviors such as damage growth and inelastic deformation. In that case, computational cost may become unacceptable.

In order to improve computational efficiency, iterative numerical methods have been intensively exploited during recent years and widely applied to various multi-phase problems. We first mention the fast Fourier transform (FFT)based iterative method developed for problems with complex microstructure [17-19]. In that framework, the solution to a heterogeneous linear or nonlinear problem can be found approximately after a series of Fourier and inverse Fourier transformation over physical fields, using the Lippmann-Schwinger form of the balance equations. In the same context, Yvonnet [20] recently developed a fast iterative scheme also based on the Lippmann-Schwinger equation (named therein as the SLS) but strictly and directly operating in the real space, in which local variables are determined by mapping through a relevant transformation array derived numerically from the associated characteristic problems. The FFT methods have been applied successfully to deal with purely elastic or elastoplastic behaviors of composites under periodic boundary conditions $[17,18,21,22]$, while the SLS method [20] has been applied so far only to linear problems. When compared to the standard finite element methods, the schemes based on the periodic LippmannSchwinger equation have evidenced the following attractive advantages.

- Discrete variables (e.g., strains, temperature) involved at material grids are computed iteratively by repeating the mapping operation with a same transformation array. This salient feature is particularly favorable for parallel programming;

- These schemes avoid completely the assembly and storage of finite element matrices and thus there is no need to solve large sparse linear systems of equations that inevitably arise in FE analyses. Therefore, computational cost and memory requirement in the Lippmann-Schwinger equation-based iterative methods are reduced significantly.

- For problems with specific boundary conditions, such as periodic ones, global stiffness matrix generated in the FEM is not positively definite, which renders, to some extent, difficulties in the numerical treatment, especially when problems to be solved are in large dimensions. On the contrary, methods based on Lippmann-Schwinger iterative schemes such as FFT [20] and SLS [17] are more robust in that case, as shown in the present work.

Although significant advances have been achieved, few efforts in the field have been dedicated so far to voxel- 
based modeling of material deterioration and strain softening induced by damage growth [23]. Indeed, unlike linear elastic or elastoplastic problems, materials suffering progressive damage can experience strongly nonlinear behaviors, including both strain hardening and softening phases. In order to capture the non-linearity of mechanical responses, external load is usually applied incrementally. Moreover, aiming at efficient implementations, constitutive equations are popularly cast into its rate (incremental) form in which a tangential operator is involved. Noticing that the abovementioned iterative schemes are implemented directly in the non-incremental form, there is a practical need to develop an increment-enhanced iterative scheme so as to coincide with the requirement by nonlinear problems.

The objective of this work is to construct an incremental-iterative scheme for image-based modelling of damage evolution in heterogeneous quasi-brittle microstructures. For this purpose, we develop in Section 2 the proposed scheme for nonlinear materials, whose general form can be applied either to Fourier (FFT) or real-space (SLS) Lippmann-Schwinger-based iterative schemes. Next, the scheme is particularized to the SLS method, i.e. the application of Lippmann-Schwinger iterative scheme in the real-space. In Section 3, the scheme is derived for heterogeneous damageable materials. For this purpose, a simple isotropic tensile damage model is formulated and the related numerical implementation details are provided. Section 4 is dedicated to validating the proposed incremental-iterative scheme by means of benchmark problems and voxel-based concrete microstructure problems obtained from microtomography images. For each test, the SLS- and FEM-based numerical predictions are compared in terms of global and local responses, as well as in term of computational efficiency.

\section{Formulation of the incremental-iterative scheme for nonlinear heterogeneous problems}

\subsection{Incremental form of elastic damage problems}

We consider a representative volume element (RVE) with the domain $\Omega \subset \mathbb{R}^{D}, D$ being the space dimension. The RVE is associated with the microstructure of a composite consisting of $r \in\{1,2, \ldots, N\}$ homogeneous phases, occupying subdomains denoted by $\Omega^{(r)}$. The interfaces between the phases are taken to be perfect for the sake of simplicity. As usually performed in micromechanics, the volume average over $\Omega$ is symbolized as $\langle\cdot\rangle_{\Omega}$. Thus, the macroscopic (or overall) strain and stress tensors, denoted respectively by $\bar{\varepsilon}$ and $\bar{\sigma}$, are defined as the volume average of the microscopic (or local) counterparts $\varepsilon(x)$ and $\sigma(x)$

$$
\bar{\varepsilon}=\langle\varepsilon(x)\rangle_{\Omega}, \bar{\sigma}=\langle\sigma(x)\rangle_{\Omega} .
$$

The problem to be solved is such that for a given strain $\overline{\boldsymbol{\varepsilon}}$, we determine the local strain solution $\boldsymbol{\varepsilon}(\boldsymbol{x})$ and the corresponding stress field $\boldsymbol{\sigma}(\boldsymbol{x})$ as well as the overall macroscopic response $\overline{\boldsymbol{\sigma}}$. In that process, the following conditions must be satisfied

$$
\begin{aligned}
& \operatorname{div} \sigma(x)=0, \quad \forall x \in \Omega, \\
& \langle\varepsilon(x)\rangle_{\Omega}=\bar{\varepsilon} .
\end{aligned}
$$


When taking into account damage effects for some phases of the RVE, the local constitutive equation in elastic damage regime can be written in the general form $\boldsymbol{\sigma}(\boldsymbol{x})=\mathbb{C}(d(\boldsymbol{x})): \boldsymbol{\varepsilon}(\boldsymbol{x})$, in which $d(\boldsymbol{x})$ is a scalar-valued damage variable used to describe any isotropic damage state at material point $\boldsymbol{x}$ and $\mathbb{C}(d(\boldsymbol{x}))$ represents the fourth-order elasticity tensor of the damaged material. Differentiation of $\boldsymbol{\sigma}(\boldsymbol{x})$ with respect to the variables $\boldsymbol{\varepsilon}$ and $d$ gives

$$
\dot{\boldsymbol{\sigma}}(\boldsymbol{x})=\left[\mathbb{C}^{\prime}(d(\boldsymbol{x})): \boldsymbol{\varepsilon}(\boldsymbol{x})\right] \dot{d}(\boldsymbol{x})+\mathbb{C}(d(\boldsymbol{x})): \dot{\boldsymbol{\varepsilon}}(\boldsymbol{x})
$$

where $\mathbb{C}^{\prime}(d(\boldsymbol{x}))$ denotes the differentiation of $\mathbb{C}(d(\boldsymbol{x}))$ with respect to the damage variable. It is known that in the classical Continuum Damage Mechanics, $\dot{d}(\boldsymbol{x})$ can be related linearly to the strain rate $\dot{\boldsymbol{\varepsilon}}(\boldsymbol{x})$ [24]. The following rate form of the constitutive equation is finally obtained

$$
\dot{\boldsymbol{\sigma}}(\boldsymbol{x})=\mathbb{C}^{\tan }(\boldsymbol{\varepsilon}(\boldsymbol{x}), d(\boldsymbol{x})): \dot{\boldsymbol{\varepsilon}}(\boldsymbol{x}),
$$

where $\mathbb{C}^{\tan }(\boldsymbol{\varepsilon}(\boldsymbol{x}), d(\boldsymbol{x}))$ is the associated tangential stiffness operator.

Based on the above formulations, an alternative balance equation in rate form is achieved

$$
\begin{aligned}
\operatorname{div} \dot{\boldsymbol{\sigma}}(\boldsymbol{x}) & =0, \quad \forall \boldsymbol{x} \in \Omega \\
\langle\dot{\boldsymbol{\varepsilon}}(\boldsymbol{x})\rangle_{\Omega} & =\dot{\overline{\boldsymbol{\varepsilon}}}
\end{aligned}
$$

In this work, we propose an incremental -iterative scheme to solve the nonlinear microscopic problem of damage evolution using the rate form (5) in tandem with a Lippmann-Schwinger iterative scheme. Remark that the above derivation procedure is also suitable for other nonlinear material models, such as elastoplastic models, coupled plasticitydamage models.

\subsection{Incremental-iterative scheme of Lippman-Schwinger type}

In this section, we develop an increment-enhanced iterative scheme for nonlinear problems in heterogeneous materials. The proposed method is based on the Lippman-Schwinger equation in terms of a stress increment.

Let first introduce the time-stepping $\mathcal{T}=\left[0, t_{1}, t_{2}, \ldots, t_{n}, t_{n+1}, \ldots, T\right]$ with $\Delta t_{n}=t_{n}-t_{n-1}$ being the $n^{\text {th }}$ time interval and $T$ final simulation time. Given $\boldsymbol{\varepsilon}^{n}(\boldsymbol{x}), d^{n}(\boldsymbol{x})$ and $\boldsymbol{\sigma}^{n}(\boldsymbol{x})$ at the beginning of time $t_{n+1}$, the aim is to find the new increments $\triangle \boldsymbol{\varepsilon}^{n+1}(\boldsymbol{x})$ and $\triangle d^{n+1}(\boldsymbol{x})$ and to update the variables $\boldsymbol{\varepsilon}^{n+1}(\boldsymbol{x})=\boldsymbol{\varepsilon}^{n}(\boldsymbol{x})+\triangle \boldsymbol{\varepsilon}^{n+1}(\boldsymbol{x}), d^{n+1}(\boldsymbol{x})=d^{n}(\boldsymbol{x})+$ $\triangle d^{n+1}(\boldsymbol{x})$ and $\boldsymbol{\sigma}^{n+1}(\boldsymbol{x})=\boldsymbol{\sigma}^{n}(\boldsymbol{x})+\Delta \boldsymbol{\sigma}^{n+1}(\boldsymbol{x})$. The stress field can then be approximated by applying Eq. (4):

$$
\boldsymbol{\sigma}^{n+1}(\boldsymbol{x})=\boldsymbol{\sigma}^{n}(\boldsymbol{x})+\mathbb{C}^{\tan }\left(\boldsymbol{\varepsilon}^{n+1}(\boldsymbol{x}), d^{n+1}(\boldsymbol{x})\right): \Delta \boldsymbol{\varepsilon}^{n+1}(\boldsymbol{x})
$$

Then, the stress increment is expressed by

$$
\Delta \boldsymbol{\sigma}(\boldsymbol{x})=\mathbb{C}^{\tan }(\boldsymbol{\varepsilon}(\boldsymbol{x}), d(\boldsymbol{x})): \Delta \boldsymbol{\varepsilon}(\boldsymbol{x})
$$

Using Eq. (5), the following equilibrating equation in terms of the stress increment can be expressed as:

$$
\operatorname{div}(\Delta \boldsymbol{\sigma}(\boldsymbol{x}))=\operatorname{div}\left(\mathbb{C}^{\tan }(\boldsymbol{x}): \Delta \boldsymbol{\varepsilon}(\boldsymbol{x})\right)=0
$$


Above, the increment index has been eliminated for the sake of clarity. We then define a fluctuation field $\Delta \tilde{\boldsymbol{\varepsilon}}(\boldsymbol{x})$ as the difference $\Delta \tilde{\boldsymbol{\varepsilon}}(\boldsymbol{x})=\Delta \boldsymbol{\varepsilon}(\boldsymbol{x})-\Delta \overline{\boldsymbol{\varepsilon}}$, which in turn allows to reformulate the field of strain increments as a sum of two terms

$$
\triangle \boldsymbol{\varepsilon}(\boldsymbol{x})=\Delta \overline{\boldsymbol{\varepsilon}}+\Delta \tilde{\boldsymbol{\varepsilon}}(\boldsymbol{x})
$$

Further, by introducing a uniform reference material with elasticity tensor $\mathbb{C}^{0}$ to rewrite $\mathbb{C}^{\tan }(\boldsymbol{x})$ as $\mathbb{C}^{\tan }(\boldsymbol{x})=$ $\mathbb{C}^{0}+\left(\mathbb{C}^{\tan }(\boldsymbol{x})-\mathbb{C}^{0}\right)$ and then defining the polarisation field $\boldsymbol{\tau}(x)=\left(\mathbb{C}^{\tan }(\boldsymbol{x})-\mathbb{C}^{0}\right): \Delta \boldsymbol{\varepsilon}(\boldsymbol{x})$, Eq. (8) is cast into the following form

$$
\operatorname{div}\left(\mathbb{C}^{0}: \Delta \tilde{\boldsymbol{\varepsilon}}(\boldsymbol{x})\right)=-\operatorname{div}(\boldsymbol{\tau}(\boldsymbol{x}))
$$

It has been shown that the solution to problem (10) can be expressed as a convolution product [17]

$$
\triangle \tilde{\boldsymbol{\varepsilon}}(\boldsymbol{x})=-\Gamma^{0} * \tau(\Delta \boldsymbol{\varepsilon}(\boldsymbol{x})),
$$

with $\Gamma^{0}$ being the associated Green operator. Substitution of Eq.(9) into Eq.(11) gives the relation

$$
\triangle \boldsymbol{\varepsilon}(\boldsymbol{x})=\Delta \bar{\varepsilon}-\Gamma^{0} * \tau(\Delta \varepsilon(x))
$$

It is then possible to construct the following iteration

$$
\triangle \boldsymbol{\varepsilon}^{r+1}(\boldsymbol{x})=\Delta \overline{\boldsymbol{\varepsilon}}-\boldsymbol{\Gamma}^{0} * \boldsymbol{\tau}\left(\triangle \boldsymbol{\varepsilon}^{r}(\boldsymbol{x})\right)
$$

where $r$ denotes the iteration index varying individually in each strain increment. Further, by making use of the property

$$
\Gamma^{0}\left(\mathbb{C}^{0}: \Delta \boldsymbol{\varepsilon}(\boldsymbol{x})\right)=\Delta \boldsymbol{\varepsilon}(\boldsymbol{x})-\triangle \overline{\boldsymbol{\varepsilon}}
$$

The following incremental-iterative scheme is finally achieved

$$
\Delta \boldsymbol{\varepsilon}^{r+1}(\boldsymbol{x})=\Delta \boldsymbol{\varepsilon}^{r}(\boldsymbol{x})+\delta \boldsymbol{\varepsilon}^{r+1}(\boldsymbol{x}),
$$

with

$$
\delta \boldsymbol{\varepsilon}^{r+1}(\boldsymbol{x})=-\boldsymbol{\Gamma}^{0} * \Delta \boldsymbol{\sigma}^{r}(\boldsymbol{x}) .
$$

It is noted that the scheme (15) should start from the uniform strain increment $\Delta \bar{\varepsilon}$ applied to periodic boundaries.

Remark that the above incremental formulations can be straightforwardly solved using the FFT method [17] by evaluating the convolution product $\boldsymbol{\Gamma}^{0} * \Delta \boldsymbol{\sigma}^{n}(\boldsymbol{x})$ in the Fourier space and applying an inverse Fourier transformation to evaluate the constitutive relation $\mathbb{C}^{\tan }(\boldsymbol{x}): \Delta \boldsymbol{\varepsilon}(\boldsymbol{x})$. In this work, we propose to use the framework developed in [20] where the convolution product is evaluated in the real space only through a transformation array. 


\subsection{Numerical discretization}

Let us consider a material structure model containing $n_{g}$ grids. Since the initial problem has been linearized, $\delta \boldsymbol{\varepsilon}(\boldsymbol{x})$ in Eq.( 16) can be evaluated by applying the principle of superposition, that is to say that for a material grid located at $\boldsymbol{x} \in \Omega$, the correction $\delta \boldsymbol{\varepsilon}(\boldsymbol{x})$ to $\Delta \boldsymbol{\varepsilon}(\boldsymbol{x})$ can be calculated by taking into account the contribution by stress increments at all material grids under consideration. For this purpose, one can perform the following discretization [20],

$$
\delta \boldsymbol{\varepsilon}_{i j}^{n+1}(\boldsymbol{x})=\sum_{p} \sum_{k} \psi_{i j, k}\left(\boldsymbol{x}, \boldsymbol{x}^{p}\right) \Delta \boldsymbol{\sigma}_{k}^{n}\left(\boldsymbol{x}^{p}\right)
$$

where $\psi_{i j, k}$ is a strain transformation (basis) matrix, which in fact plays the role of weighting coefficients in computing the convolution product. The determination of $\psi_{i j, k}$ consists in solving a boundary value eigenstress problems in a small subdomain $\Omega^{*}$ around one voxel $\mathbf{x}^{p}$ in a homogeneous reference medium. The value range of the subscript $k$ in $\psi_{i j, k}$ depends on the problem to be handled. Precisely, $k$ ranges from 1 to 3 for 2D problems and ranges from 1 to 6 for $3 \mathrm{D}$ problems.

For example, in the 2D case, there exists the following decomposition upon any plane stress increment $\Delta \boldsymbol{\sigma}\left(\boldsymbol{x}^{p}\right)$ defined at grid $\boldsymbol{x}^{p}, p$ ranging from 1 to $n_{g}$

$$
\Delta \boldsymbol{\sigma}\left(\boldsymbol{x}^{p}\right)=\Delta \sigma_{11}\left(\boldsymbol{x}^{p}\right)\left[\begin{array}{ll}
1 & 0 \\
0 & 0
\end{array}\right]+\Delta \sigma_{12}\left(\boldsymbol{x}^{p}\right)\left[\begin{array}{ll}
0 & 1 \\
1 & 0
\end{array}\right]+\Delta \sigma_{22}\left(\boldsymbol{x}^{p}\right)\left[\begin{array}{ll}
0 & 0 \\
0 & 1
\end{array}\right],
$$

from which is derived three bases common to all local stresses measured at material grids

$$
\mathcal{S}_{1}=\left[\begin{array}{ll}
1 & 0 \\
0 & 0
\end{array}\right], \mathcal{S}_{2}=\left[\begin{array}{ll}
0 & 1 \\
1 & 0
\end{array}\right], \mathcal{S}_{3}=\left[\begin{array}{ll}
0 & 0 \\
0 & 1
\end{array}\right]
$$

When adopting the notations

$$
\Delta \sigma_{1}=\Delta \sigma_{11}\left(\boldsymbol{x}^{p}\right), \Delta \sigma_{2}=\Delta \sigma_{12}\left(\boldsymbol{x}^{p}\right), \Delta \sigma_{3}=\Delta \sigma_{22}\left(\boldsymbol{x}^{p}\right)
$$

the basis $\mathcal{S}_{k}$ will be related to the respective stress components $\Delta \sigma_{k}, k=1,2,3$.

Furthermore, as the reference medium is homogeneous, and assumed to be isotropic, the transformation matrix $\psi_{i j, 3}$ associated with the basis $\mathcal{S}_{3}$ can be derived from $\psi_{i j, 1}$ by a simple rotation. Thus, in order to construct the transformation array $\psi_{i j, k}$, we only need to solve the following two characteristic problems

$$
\psi_{, k}(\boldsymbol{x})=-\Gamma^{0} * \mathcal{S}_{k}, \quad k=1,2 .
$$

When boundary conditions are such that $\psi_{, k}(\boldsymbol{x})=0$, it is proved that problem (21) is equivalent to the following form

$$
\operatorname{div}\left(\mathbb{C}^{0}: \psi_{, k}(\boldsymbol{x})\right)=-\operatorname{div}\left(\mathcal{S}_{k}\right), \quad k=1,2, \forall \mathbf{x} \in \Omega^{*},
$$


where $\Omega^{*}$ is a small domain surrounding a pixel $\mathbf{x}^{p}$. The problem (22) is solved over $\Omega^{*}$ with zero Dirichlet boundary conditions prescribed in the boundary of $\Omega^{*}$. For more details on the relevant weak formulations, one can refer to the work by Yvonnet [20].

\section{Application to damaged heterogeneous materials}

We now proceed to present a simple isotropic damage model to describe elastic damage in some phases of the elementary volume. Emphasize that the application of the proposed method is not restricted to isotropic damage evolution problems but applicable to more complex nonlinear models. For simplicity, a scale-valued damage variable $d$ is introduced to describe the state of isotropic material degradation. The damage is evaluated by a linear combination of the tension- and compression-related parts. Numerical implementations within the incremental-iterative scheme will also be discussed.

\subsection{A simple elastic damage model}

The elasticity tensor of the damaged material is simply formulated as [25]

$$
\mathbb{C}(\boldsymbol{x})=(1-d(\boldsymbol{x})) \mathbb{C}^{s}(\boldsymbol{x}),
$$

where $\mathbb{C}^{s}(\boldsymbol{x})$ is the elasticity tensor of the material in its undamaged state and $d(\boldsymbol{x})$ the damage field. Phenomenologically, the damage level can be related to the equivalent tensile strain. The latter is defined by

$$
\varepsilon_{e q t}=\left\|\left\langle\varepsilon_{i}\right\rangle_{+}\right\| .
$$

Above, $\varepsilon_{i}$ are the principle strains. $\langle\cdot\rangle_{+}$is the Macaulay-type operation, i.e. for any real variable $x,\langle x\rangle_{+}=x$ when $x \geq 0$ and 0 , otherwise.

In order to reflect the asymmetry of material strength in tension and in compression, we distinguish the traction effect and compression effect on damage evolution

$$
\begin{aligned}
& d_{t}=c_{t}\left[1-\exp \left(-B_{t}\left\langle\varepsilon_{e q t}-\kappa_{0}\right\rangle\right)\right], \\
& d_{c}=c_{c}\left[1-\exp \left(-B_{c}\left\langle\varepsilon_{e q t}-\kappa_{0}\right\rangle\right)\right] .
\end{aligned}
$$

Above, $c_{t}$ and $c_{c}$ represent the critical damage values respectively for tensile and compressive loadings. $B_{t}$ and $B_{c}$ are two model's parameters used to control the rate of damage evolution. Moreover, $\kappa_{0}$ denotes the initial threshold for damage evolution. Based on these formulations, the damage at a given equivalent strain is calculated by combining the two parts

$$
d=(1-\xi) d_{c}+\xi d_{t}
$$

and the weighting coefficient $\xi$ is related to the principal stresses $\sigma_{i}$

$$
\xi=\frac{\left\|\left\langle\sigma_{i}\right\rangle_{+}\right\|}{\left\|\sigma_{i}\right\|} .
$$


It is easily seen that $\xi=1$ for uniaxial tension whereas $\xi=0$ for uniaxial compression.

In order to derive the tangential operator $\mathbb{C}^{\tan }(\boldsymbol{x})$, we can introduce the fourth-order projection tensor $\mathbb{P}^{+}$in [26] so as that the equivalent tensile $\varepsilon_{e q t}$ is related explicitly to the strain tensor, such that $\varepsilon_{e q t}=\sqrt{\varepsilon: \mathbb{P}^{+}: \varepsilon}$, which allows to write the stress-strain relation in its rate form.

The tangential operator $\mathbb{C}^{\tan }(\boldsymbol{x})$ is generally more complex than $\mathbb{C}(\boldsymbol{x})$, which would be an apparent disadvantage from the viewpoint of parallel programming. For the sake of simplicity, we then approximate in the present work $\mathbb{C}^{\tan }(\boldsymbol{x})$ by $\mathbb{C}(\boldsymbol{x})$ in both the finite element calculations and the proposed incremental iterations.

\subsection{Implementation details}

In order to guarantee a sufficient precision for both the stress and strain field, a combining error indicator is introduced, as described in Eq. (33). A tolerance value $\epsilon=10^{-3}$ has been used in all the numerical tests. Before the computation, for a chosen $\mathbb{C}^{0}$, we construct the strain transformation array $\psi$. At the end of $n^{\text {th }}$ loading increment, the fields $\boldsymbol{\sigma}^{n}(\boldsymbol{x}), \boldsymbol{\varepsilon}^{n}(\boldsymbol{x}), d^{n}(\boldsymbol{x})$ being assumed to be completely determined by calculating iteratively the strain increment $\triangle \boldsymbol{\varepsilon}^{n}(\boldsymbol{x})$, implementation details for the $(n+1)^{\text {th }}$ loading step are described as follows:

1. Initialize the field of the strain increment $\Delta \boldsymbol{\varepsilon}^{n+1}(\boldsymbol{x})=\Delta \overline{\boldsymbol{\varepsilon}}^{n}$ and predict $\Delta \boldsymbol{\sigma}^{n+1,0}(\boldsymbol{x})=\mathbb{C}\left(d^{n}(\boldsymbol{x})\right): \Delta \boldsymbol{\varepsilon}^{n+1}(\boldsymbol{x})$. Impose the initial values $r=0$ and set $\ell=1$

- While $\ell>\epsilon$

2. Calculate the modification to $\triangle \boldsymbol{\varepsilon}^{n+1}(\boldsymbol{x})$

$$
\delta \varepsilon_{i j}^{n+1, r+1}(\boldsymbol{x})=\sum_{p} \sum_{k} \psi_{i j, k}\left(\boldsymbol{x}, \boldsymbol{x}^{p}\right) \Delta \sigma_{k}^{n+1, r}\left(\boldsymbol{x}^{p}\right)
$$

3. Update the strain increment

$$
\Delta \boldsymbol{\varepsilon}^{n+1}(\boldsymbol{x})=\Delta \boldsymbol{\varepsilon}^{n+1}(\boldsymbol{x})+\delta \boldsymbol{\varepsilon}^{n+1, r+1}(\boldsymbol{x})
$$

leading to the result

$$
\boldsymbol{\varepsilon}^{n+1}(\boldsymbol{x})=\boldsymbol{\varepsilon}^{n}(\boldsymbol{x})+\Delta \boldsymbol{\varepsilon}^{n+1}(\boldsymbol{x})
$$

4. Determine the field of damage $d^{n+1}(\boldsymbol{x})$

5. Calculate the stress increments at all material grids

$$
\Delta \boldsymbol{\sigma}^{n+1, r+1}(\boldsymbol{x})=\mathbb{C}\left(d^{n+1}(\boldsymbol{x})\right): \Delta \boldsymbol{\varepsilon}^{n+1}(\boldsymbol{x})
$$

6. Compute the convergence indicator

$$
r=r+1
$$

$$
\ell=\max \left(\frac{\left\|\delta \boldsymbol{\varepsilon}^{n+1, r+1}(\boldsymbol{x})\right\|}{\left\|\Delta \boldsymbol{\varepsilon}^{n+1}(\boldsymbol{x})\right\|}, \frac{\left\|\Delta \boldsymbol{\sigma}^{n+1, r+1}(\boldsymbol{x})-\Delta \boldsymbol{\sigma}^{n+1, r}(\boldsymbol{x})\right\|}{\left\|\Delta \boldsymbol{\sigma}^{n+1, r}(\boldsymbol{x})\right\|}\right)
$$

\section{- End of iterations}

7. update for $(n+1)$ step

$$
\boldsymbol{\sigma}^{n+1}(\boldsymbol{x})=\mathbb{C}\left(d^{n+1}(\boldsymbol{x})\right): \boldsymbol{\varepsilon}^{n+1}(\boldsymbol{x}) .
$$




\section{Numerical examples}

In this part, numerical tests are performed to assess the ability of the proposed method in predicting material damage evolution in heterogeneous materials. For all the tests, the plane strain condition and periodic boundary conditions are assumed. Emphases here will be put on comparisons between the results obtained by the proposed method and those predicted by the standard finite element method. For simplicity, only damage induced by local tensile strains is taken into account, that is to say, the weighting coefficient $\xi$ is set to be 1 . Moreover, only the matrix is assumed damageable. The values of the parameters involved in the damage model are such that $B_{t}=10, \kappa_{0}=0.25$ and $c_{t}=0.99$. For each test, we will depict and compare the stress-strain curves and the damage configurations. The analyses presented below are strain-controlled: the total macroscopic strain is prescribed and applied incrementally on the boundary while the resulting macroscopic stress is calculated by taking the average of the local values over material grids.

\subsection{A benchmark study}

Let us consider a composite material in which the matrix phase is reinforced by long fibers of a same section and periodically arranged along a hexagonal array. We propose to perform some tests in the $2 \mathrm{D}$ context. The unit cell extracted from the structure is depicted in Figure 1. The rectangular cell taken as the RVE is composed of a matrix phase and cylindrical inclusions. Their mechanical behaviors are assumed to be isotropic, even in damaged states for the matrix. The initial values of the elastic moduli of the matrix are such that $\lambda_{\text {mat }}=1 \mathrm{MPa}$ and $\mu_{\text {mat }}=1 \mathrm{MPa}$. The width and height of the cell are denoted respectively by $b$ and $h$ and the radius of the circular inclusions by $r$, as illustrated in Figure 1. In the present and next sections, we take $b=1.725 \mathrm{~mm}, h=1 \mathrm{~mm}$ and $r=0.2 \mathrm{~mm}$. The corresponding inclusion volume fraction, denoted by $\phi_{1}$ is about $\phi_{1}=14.57 \%$. For numerical analyses, the RVE is discretized by $138 \times 80$ pixels, providing material grids of the same number and each pixel serving as an element in the FE analyses. The mapping grids are such that $n_{x} \times n_{y}=59 \times 59$ with its center located at a current material grid.

The RVE is subjected on its boundary to a macroscopic strain whose components are $\bar{\varepsilon}_{x x}=0.5$, $\bar{\varepsilon}_{y y}=0$ and $\bar{\varepsilon}_{x y}=\bar{\varepsilon}_{y x}=0$. To capture nonlinear behaviors of the material, the total strain $\bar{\varepsilon}$ is applied incrementally by 100 steps. For each step, the strain field (local strains at material grids) is determined iteratively and the local stresses are computed by the local constitutive law.

\subsubsection{Case of rigid inclusions}

In this case, the two Lamé's constants of the rigid inclusions are taken as $\lambda_{\mathrm{inc}}=10 \mathrm{MPa}$ and $\mu_{\mathrm{inc}}=10 \mathrm{MPa}$, which means ten times of the counterpart for the matrix. As for the reference isotropic material, we chose $\lambda_{\text {ref }}=6$ $\mathrm{MPa}$ and $\mu_{\text {ref }}=6 \mathrm{MPa}$. In other words, $E_{\text {ref }}=0.6 E_{\mathrm{inc}}$ and $v_{\mathrm{ref}}=v_{\mathrm{inc}}$ [21]. It is seen from Figure 2 that a very good agreement is found between the global stress-strain curves predicted by the finite element method and the proposed incremental-iterative method. The material shows a linearly elastic behaviors at the first stage of loading. It follows a hardening phase and then a strain softening phase. The nonlinearity in the stress-strain curves is completely attributed 


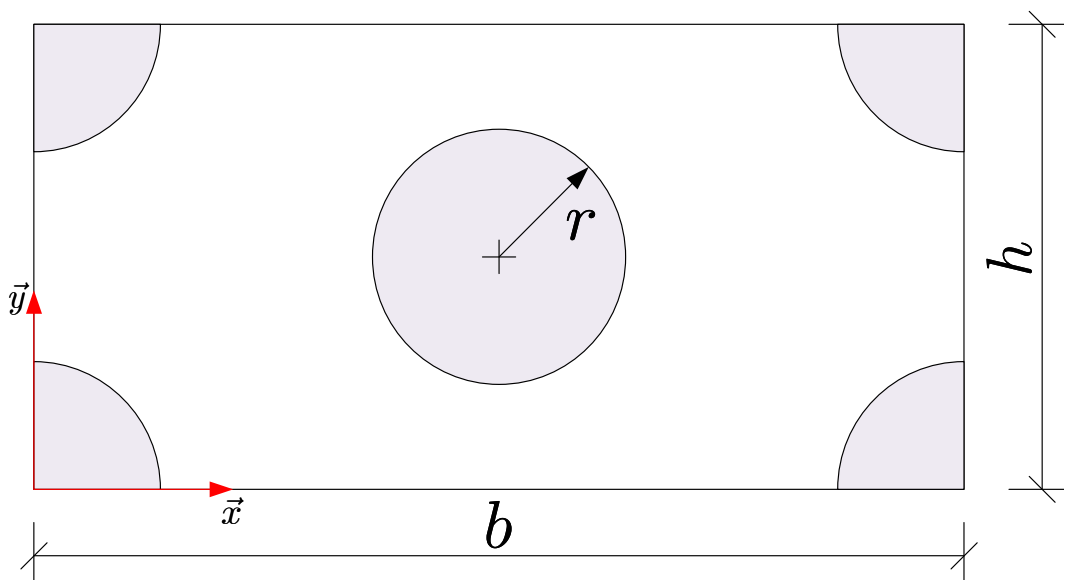

Figure 1: Geometric illustration of the benchmark structure used as the Representative Elementary Volume

to damage growth. More precisely, damage nucleates at matrix-inclusions interfaces and propagates preferentially in the loading direction. At the final stage, the coalescence of damage takes place, leading to strain softening. In Figure 3 are shown the damage distributions predicted by the standard FEM and the incremental-iterative method at the 80th loading increment, corresponding to $\bar{\varepsilon}_{x x}=0.4$. It is concluded that in the considered case the proposed iterative numerical technique can simulate the process of damage propagation and strain localization with good accuracy.

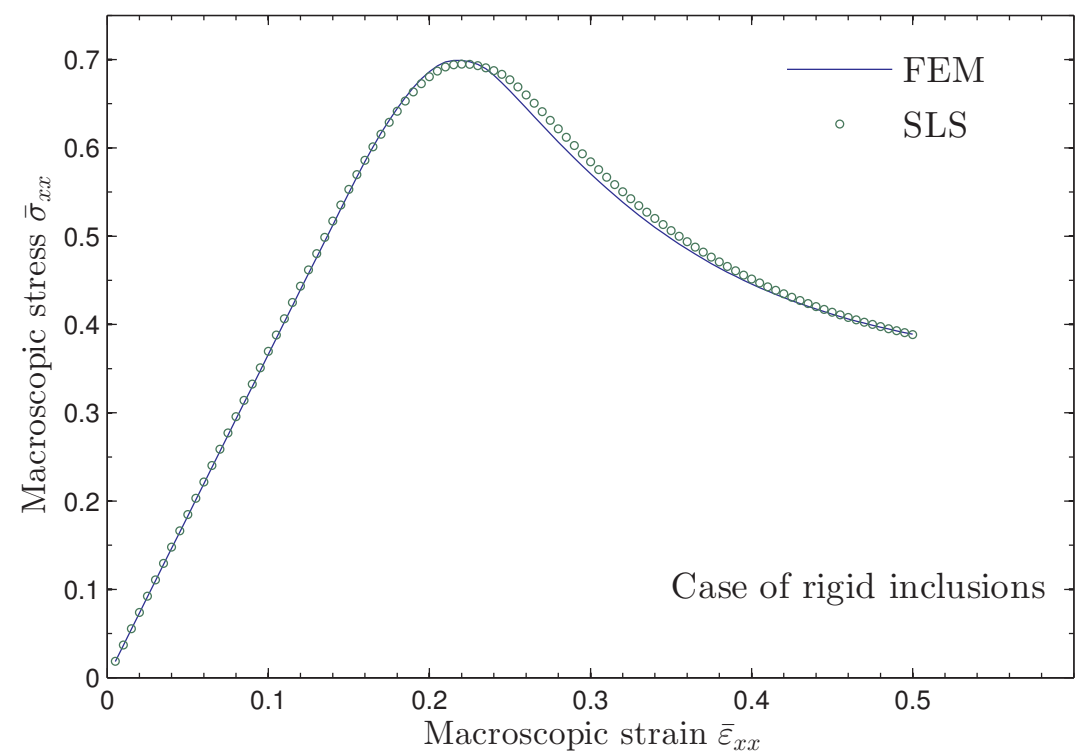

Figure 2: Comparisons between the stress-strain curves predicted by the FEM and the present method: case of regularly-distributed rigid inclusions. 

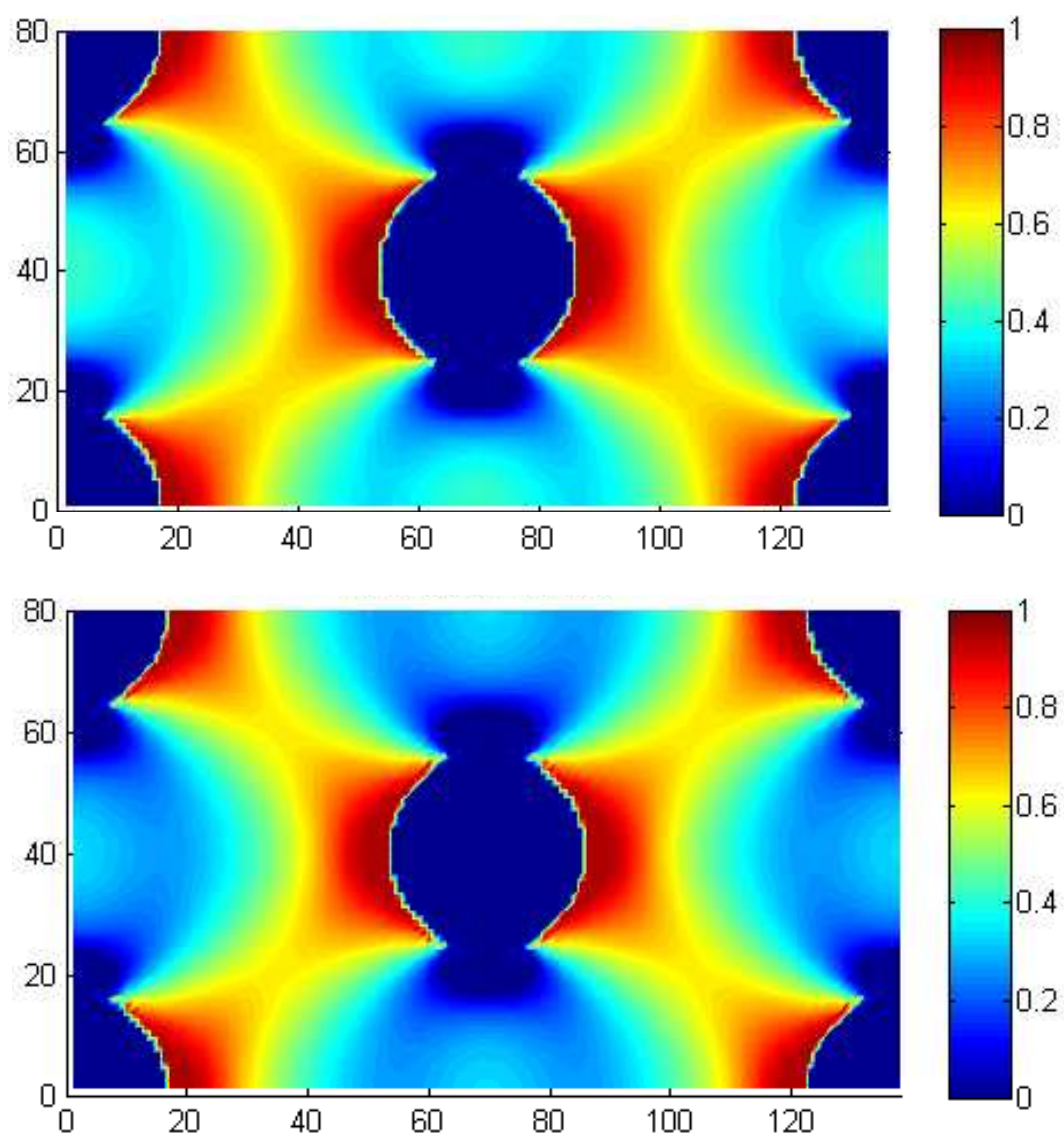

Figure 3: Comparisons on the damage distributions predicted by the FEM (upper figure) and the present method (lower figure): case of regularlydistributed rigid inclusions.

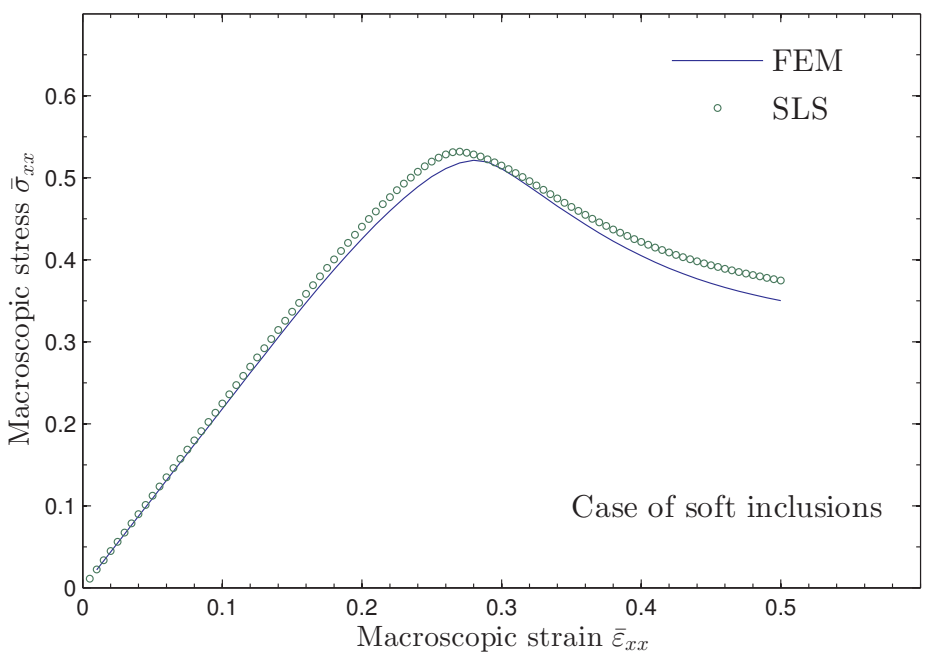

Figure 4: Comparisons between the stress-strain curves predicted by the FEM and the present method: case of regularly-distributed soft inclusions. 


\subsubsection{Case of soft inclusions}

For the case of soft inclusions, we take $\lambda_{\text {inc }}=0.1 \mathrm{MPa}$ and $\mu_{\mathrm{inc}}=0.1 \mathrm{MPa}$ for inclusions and accordingly, $\lambda_{\text {ref }}=$ $0.6 \mathrm{MPa}$ and $\mu_{\text {ref }}=0.6 \mathrm{MPa}$ for the reference isotropic material. The stress-strain curves predicted by the FEM and the present method are reported and compared in Figure 4. We notice a slight difference between the two methods for the considered size of inclusions. The diagrams of damage distribution reported in Figure 5 may explain to some extent the source of this difference. It is seen that damage coalescence predicted by the FEM is earlier than that by the present method. On this aspect, further investigations particularly on mesh dependence are still required.
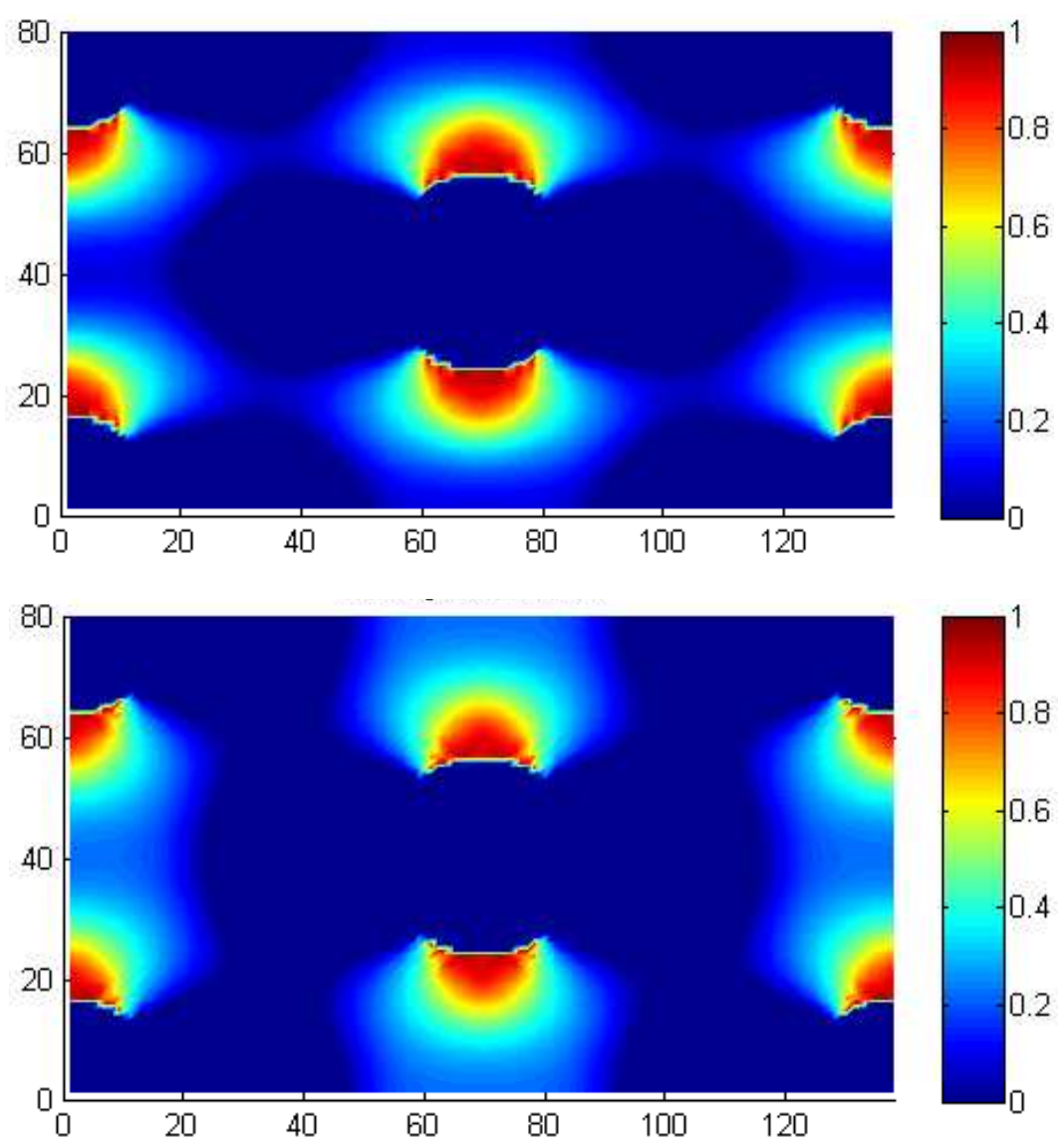

Figure 5: Comparisons on the damage distributions predicted by the FEM (upper figure) and the SLS method (lower figure): case of soft regularlydistributed inclusions.

\subsection{Tests with inclusions randomly distributed in the matrix}

In the second series of numerical tests, the RVE is still of the dimensions $b \times h=1.725 \mathrm{~mm} \times 1 \mathrm{~mm}$ and uniformly discretized by $138 \times 80$ pixels. The composite is now composed of the matrix phase and 25 randomly distributed circular inclusions. For simplicity, all the inclusions have the same radius $r=0.04 \mathrm{~mm}$, as shown in Figure 6 . In this case, the volume fraction of the inclusions is such that $\phi_{2}=0.5 \phi_{1}$. The same testing conditions described in Section 


\section{1 are adopted.}

For the case of soft inclusions, the stress-strain curves predicted by the FEM and the SLS method are depicted and compared in Figure 7. The diagrams of damage distribution at $\bar{\varepsilon}_{11}=0.4$ are reported in Figure 8. Again, there is a very good agreement between the two methods for both the global response and the damage distribution. For the case of rigid inclusions, the mechanical responses is presented in Figure 9 and the damage distributions in 10. The same conclusions as those in the case of soft inclusions can be drawn, which confirms the predictive capacity of the proposed incremental-iterative scheme.

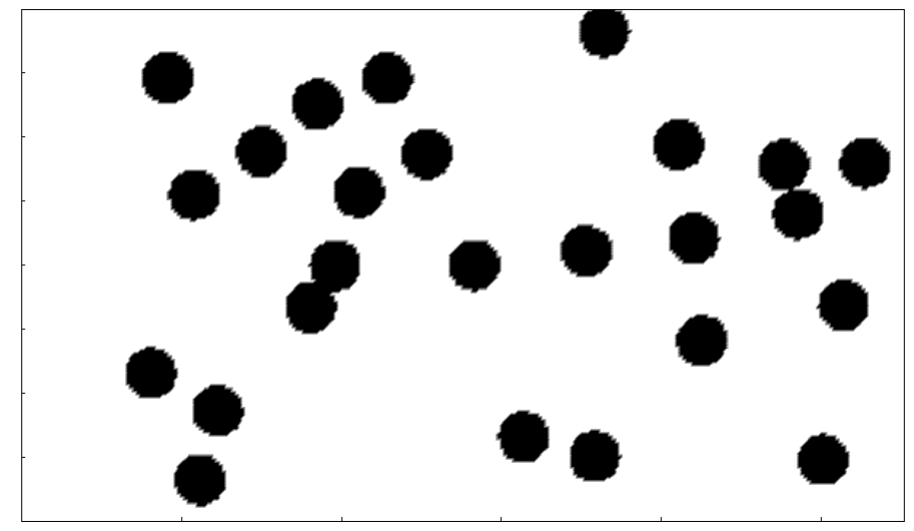

Figure 6: The microstructure of the RVE containing 25 randomly-distributed circular inclusions.

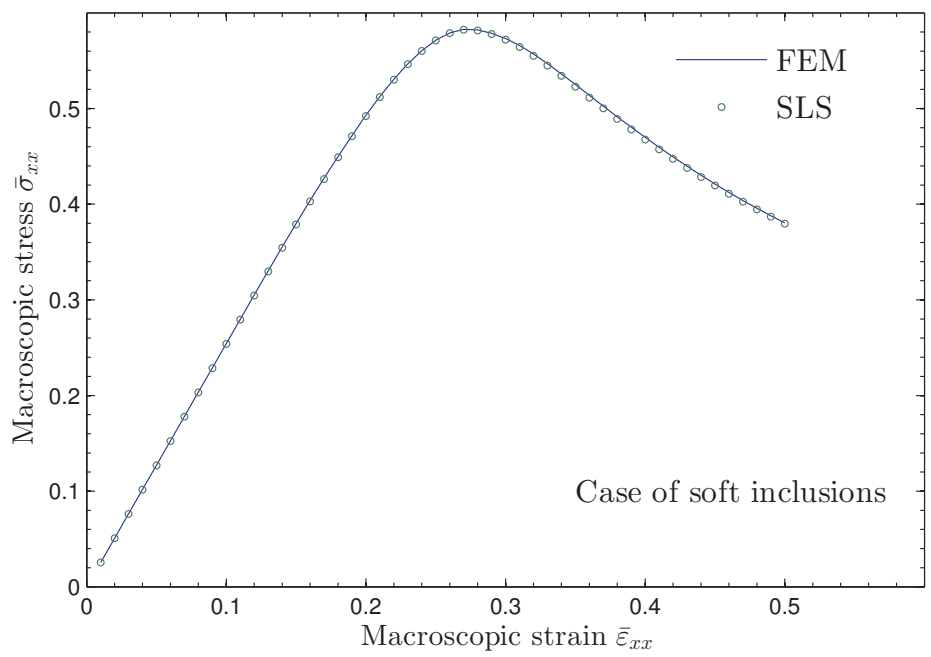

Figure 7: Comparisons on the stress-strain curves obtained by the FEM and the present method: case of randomly-distributed soft inclusions. 

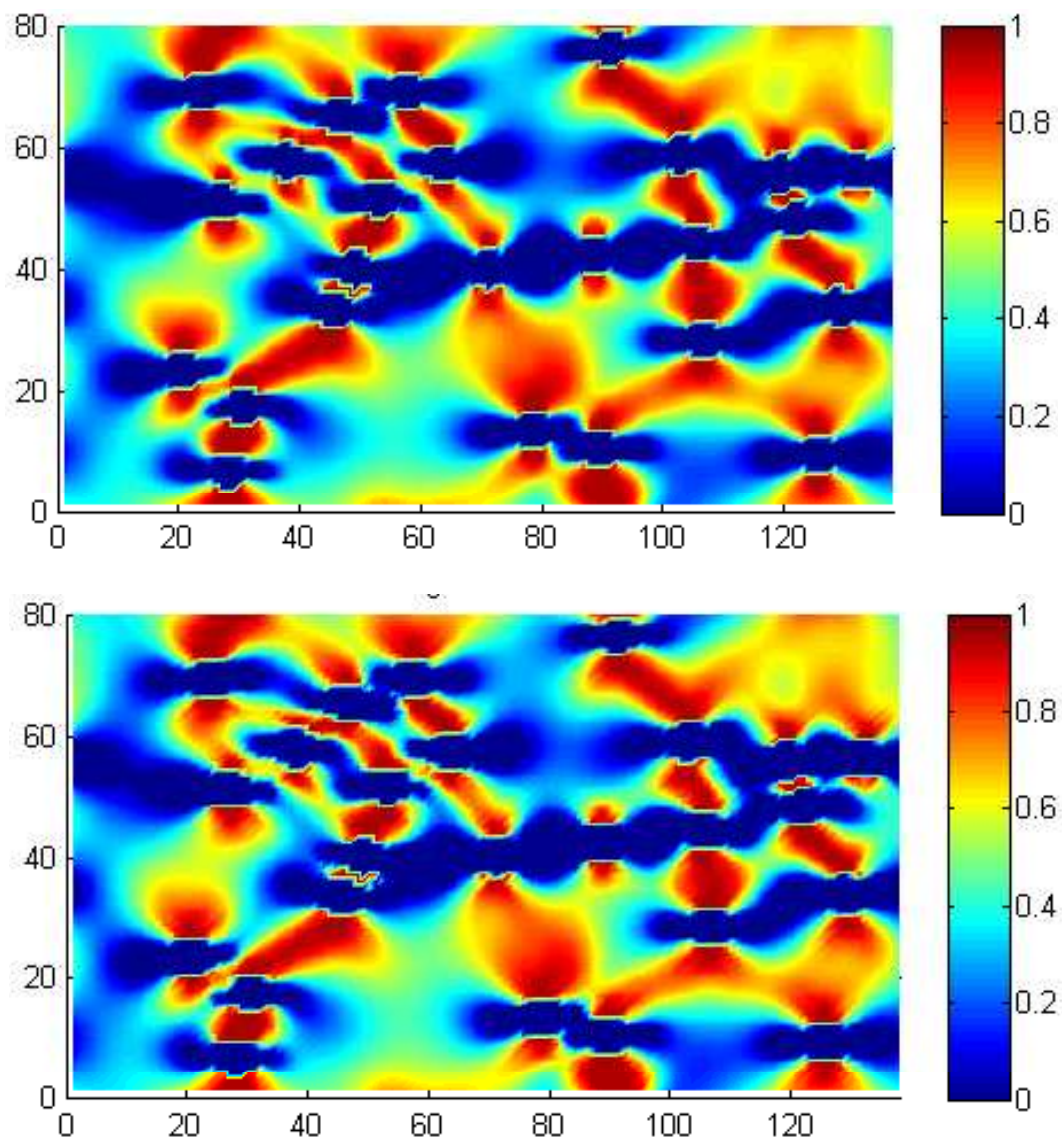

Figure 8: Damage distributions obtained by the FEM (upper figure) and the proposed incremental-iterative method (lower figure): case of randomlydistributed soft inclusions.

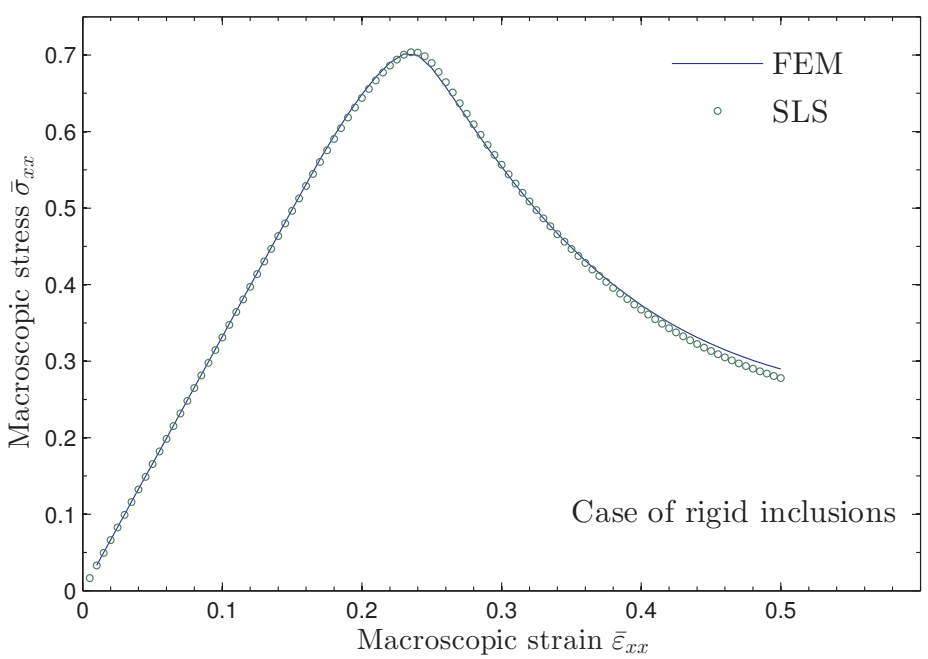

Figure 9: Comparisons on the stress-strain curves obtained by the FEM and the proposed method: case of randomly-distributed rigid inclusions. 

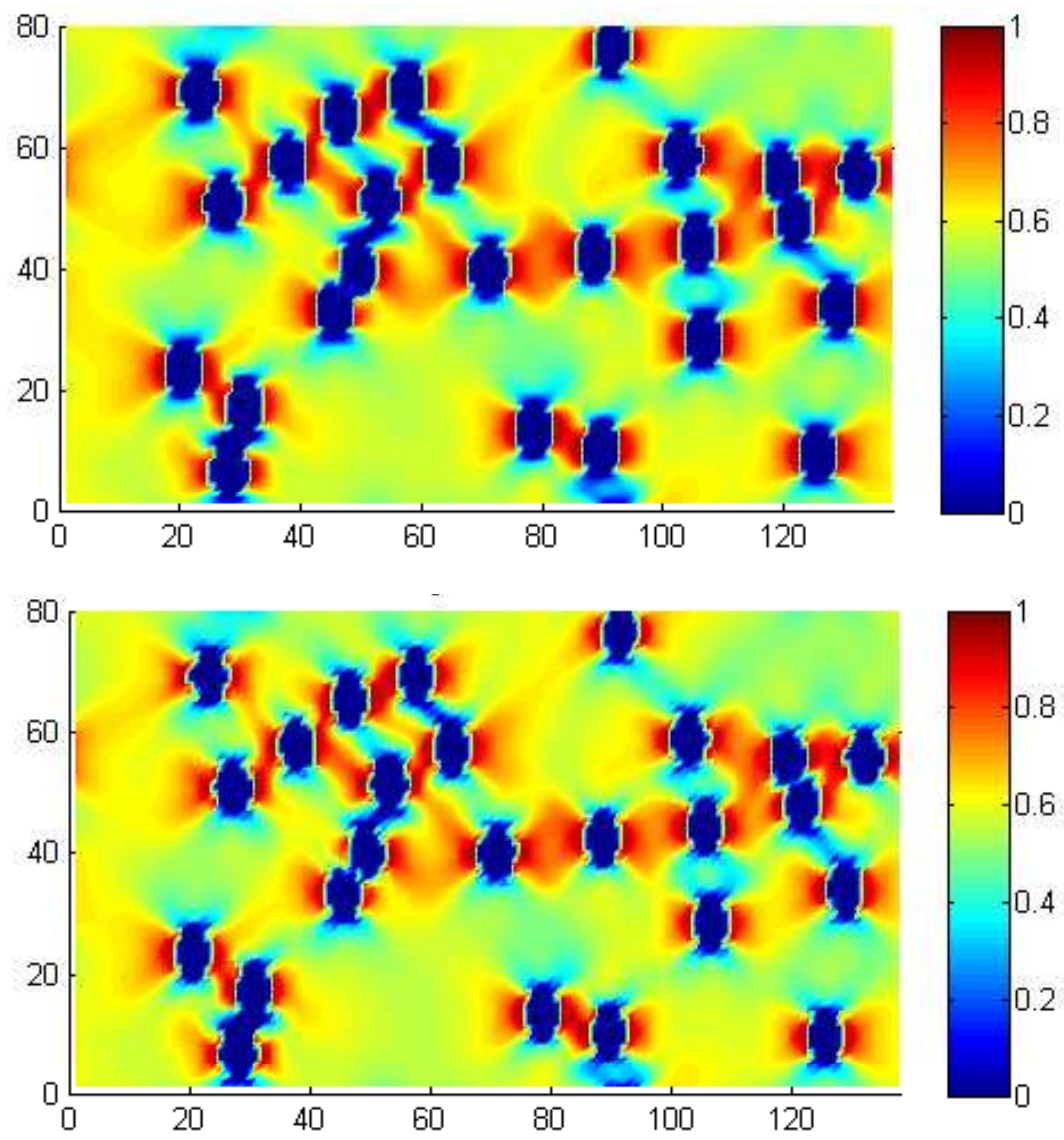

Figure 10: Comparisons on damage distributions obtained by the FEM (upper figure) and the proposed method (lower figure): case of randomlydistributed rigid inclusions.

\subsection{Damage modelling based on microtomographic data}

The last numerical tests are devoted to simulating the mechanical behaviors of a concrete sample whose microstructure and material properties in its undamaged state are obtained by microtomography. The material mainly consists of three phases: the cement-based matrix, aggregates and the voids, as described in Figure 11. According to experimental data, the aggregates are forty times stiffer than the matrix. Since the objective here is to assess the predicting ability of the incremental-iterative method, we still take $\lambda_{\text {mat }}=1 \mathrm{MPa}$ and $\mu_{\mathrm{mat}}=1 \mathrm{MPa}$ for the matrix and thus $\lambda_{\text {inc }}=40 \mathrm{MPa}$ and $\mu_{\mathrm{inc}}=40 \mathrm{MPa}$ for the aggregates. To avoid numerical difficulties related to the system of linear equations in FEM analysis, we used a small value for the elastic constants of voids such that $\lambda_{\text {void }}=10^{-3} \mathrm{MPa}$ and $\mu_{\text {void }}=10^{-3} \mathrm{MPa}$.

Microtomographic data is stored as a $150 \times 125 \times 151$ array. We take here the 75 th slice as the material and structure to be modeled. Thus, the rectangular cell contains $150 \times 125$ material grids. The curves of the global stress versus global strain is reported and compared in Figure 12. It is seen that the results predicted by the FEM and the proposed method coincides quite well with each other in the pre-peak phase and at the peak stress. In the post-peak phase, 


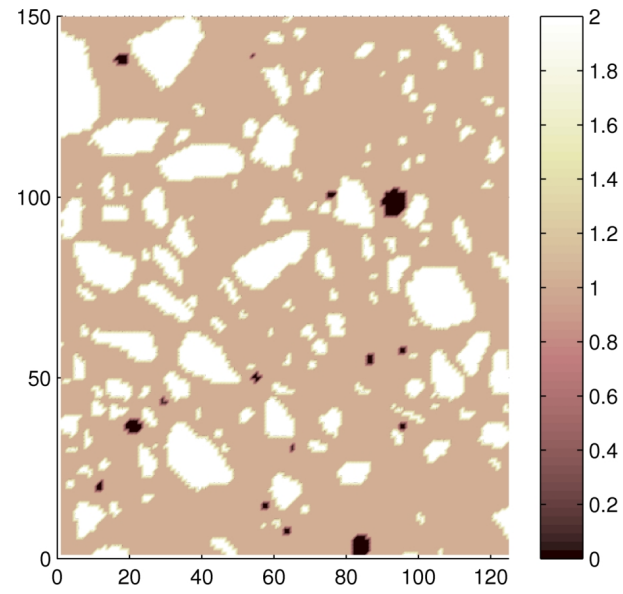

(a) model with $125 \times 150$ grids

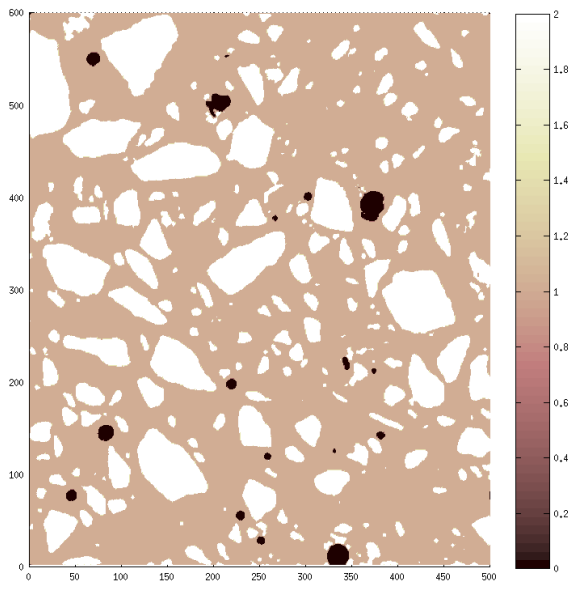

(b) model with $500 \times 600$ grids

Figure 11: Material structure of a slice of a concrete sample obtained by image processing technique. According to the color bar, value 1 stands for the cement matrix phase, 0 for voids and 2 for aggregates.

the SLS method provides a more pronounced strain softening behavior. The diagrams of damage distribution at three loading steps are presented in Figure 13 for the SLS-based method and Figure 14 for the FE analyses. Comparisons between the two methods are quite satisfactory. It is noted that damage nucleates at aggregates' corners because stress concentration there is more pronounced and the saturation of the damage criterion is more earlier. As the tensile load is increased progressively, the propagation and coalescence of diffuse damage is also predicted correctly.

We are then concerned with the influence of the choice of material slices on the mechanical behaviors. To this end, five levels of material grids are taken into account, each level providing a specific material microstructure. Next, the proposed incremental-iterative method is applied to each set of material grids. The results are plotted and compared in Figure 15. It is seen that although the five material cases lead to a globally stable mechanical response, more or less perturbations are captured in all the phases. It means that the size of the testing samples is not big enough to be taken as a representative elementary volume of the concrete under study.

\subsection{Discussions on the choice on reference materials}

Numerical experiences have evidenced that the reference material plays a critical role in the FFT-based and SLSbased computations. According to Michel et al. [21], for the isotropic linear elastic reference medium $\mathbb{C}^{0}$, a sufficient condition to ensure numerical convergence is such that

$$
k_{r e f}>\frac{1}{2} \max \{k(\boldsymbol{x})\}, \mu_{r e f}>\frac{1}{2} \max \{\mu(\boldsymbol{x})\}
$$

where $k_{r e f}$ and $\mu_{r e f}$ denote the bulk and shear moduli of the reference material, respectively.

In the present framework, some phases of heterogeneous materials will experience increasing damage and the relevant elastic moduli will be degraded. We are now interested in testing the effect of material degradation on the 


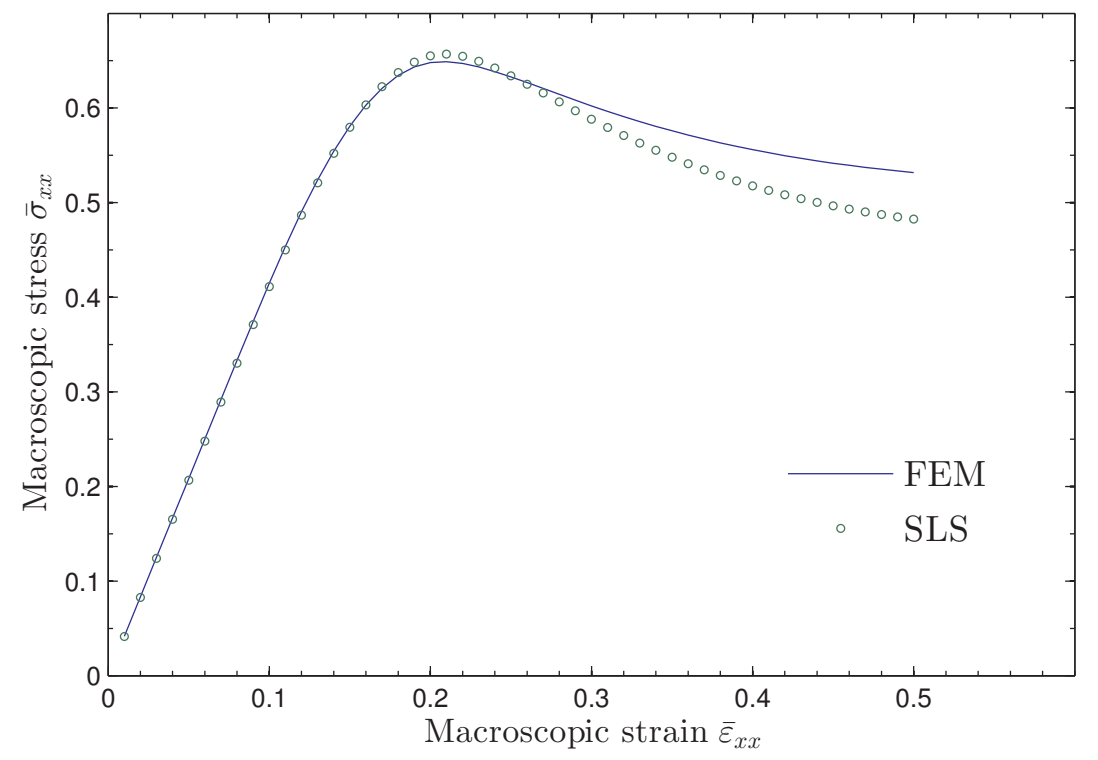

Figure 12: Comparisons on the stress-strain curves obtained by the FEM (upper figure) and the SLS method (lower figure): structure data from microtomograhic image processing technique.

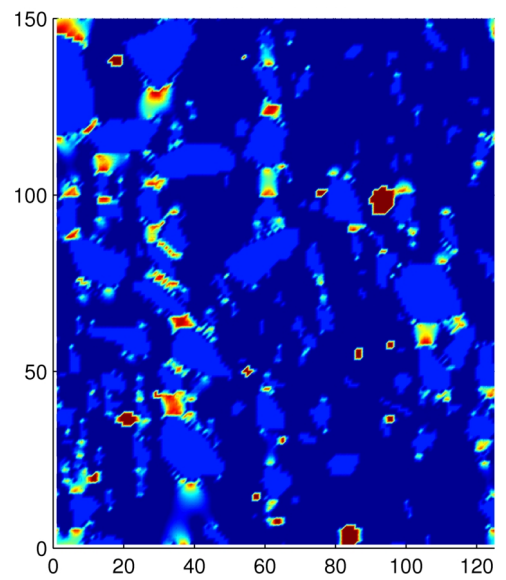

(a) 30th loading step

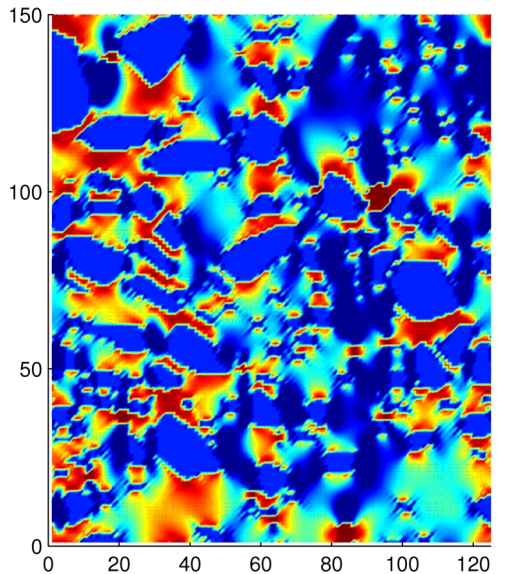

(b) 50th loading step

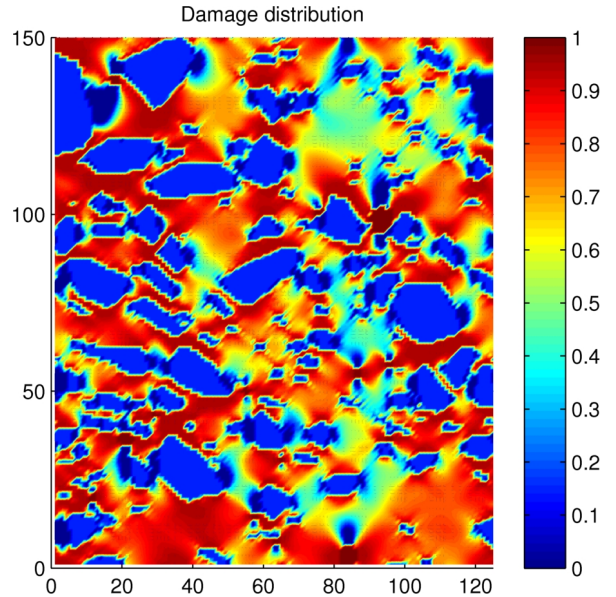

(c) 70th loading step

Figure 13: Voxel-based modelling of damage process in a slice of a concrete sample: damage distributions at three loading levels predicted by the incremental-iterative method. 


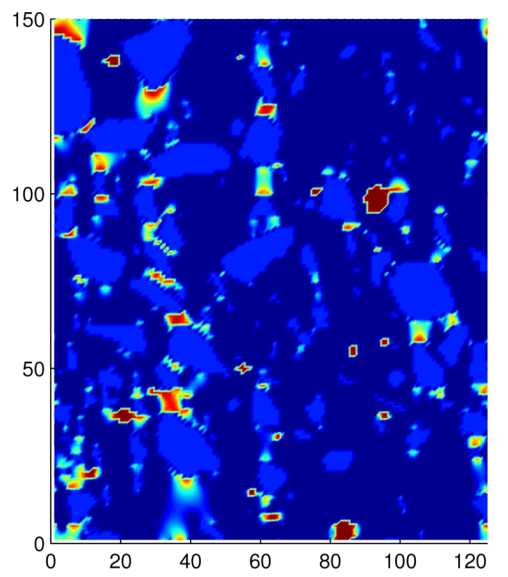

(a) 30th loading step

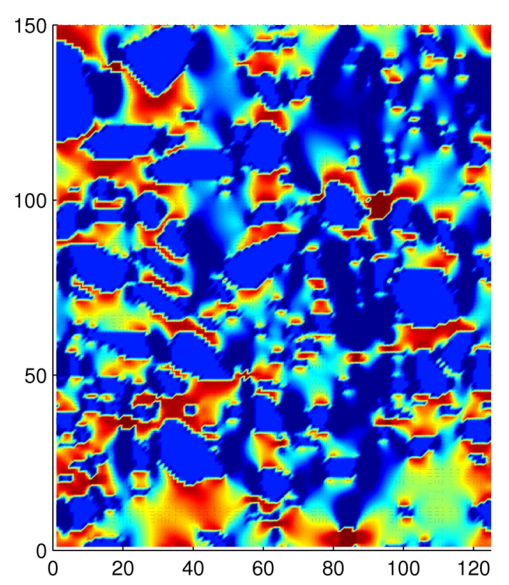

(b) 50th loading step

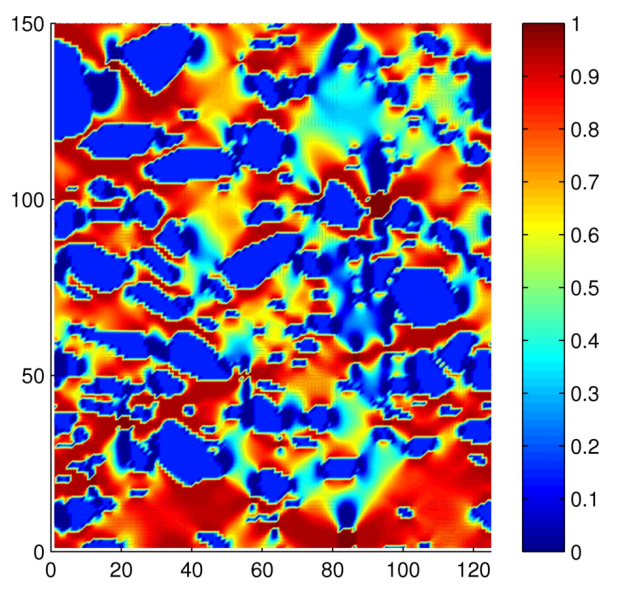

(c) 70th loading step

Figure 14: Voxel-based modelling of damage process in a slice of a concrete sample: damage distributions predicted by the FEM.

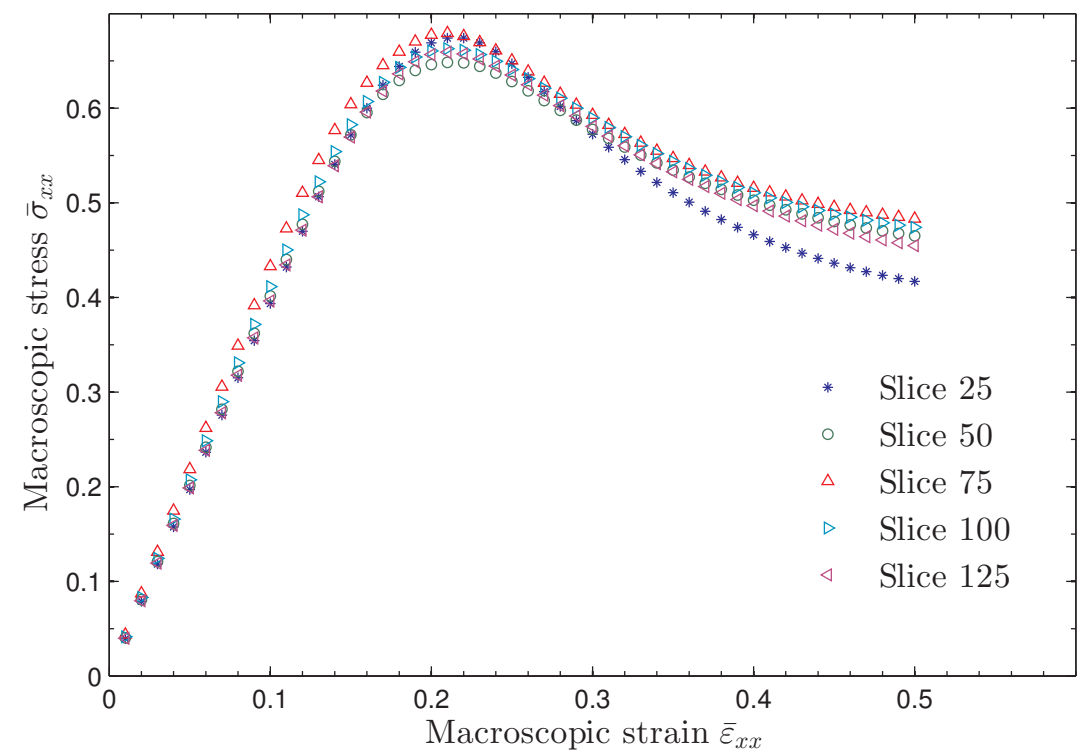

Figure 15: Comparisons between mechanical responses for 5 levels of material grids of a concrete. 
choice of a reference material. To this end, the global response with damage evolution and computational iteration numbers for the prescribed tolerance are studied by varying the values of reference material moduli. For simplicity, we take the microstructure and material elastic moduli already used in Section 4.3. By defining $\lambda_{\text {ref }}=\rho \lambda_{\text {inc }}$ and $\mu_{\text {ref }}=\rho \mu_{\text {inc }}$, numerical tests are carried out by varying the value of the ratio $\rho$. More precisely, seven tests are performed with $\rho=0.52,0.6,0.7,0.8,0.9,1.0$ and 1.5 , respectively. The predicted mechanical responses are presented and compared in Figure 16 and the iteration numbers at each loading increment are reported in Figure 17 for all the seven tests.

It is seen that the global stress-strain curve is quite stable as the reference material changes with $\rho$ ranging from 0.52 to 1.50 . Moreover, on each loading level, the number of computational iterations is slightly influenced by the choice of the ratio $\rho$ as long as it is located between the bounds 0.5 and 1.0. It is worthy indicating that numerical convergence was not achieved with $\rho=0.5$. It is concluded from the above analyses that constant values for both $k_{\text {ref }}$ and $\mu_{\text {ref }}$ are sufficient for numerical tests even involving damage evolution.

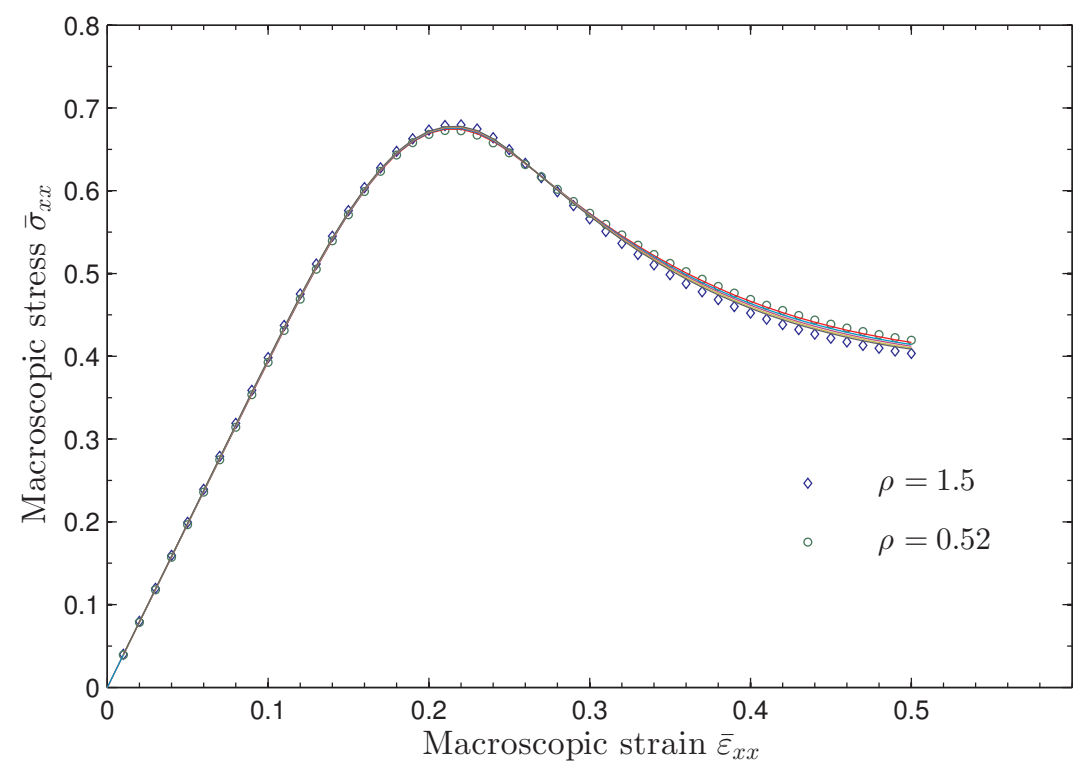

Figure 16: Comparisons on mechanical responses predicted with different values of the ratio $\rho$

\subsection{Computational efficiency of the proposed method}

We now evaluate computational efficiency of the proposed incremental iterative scheme by comparing with the standard finite element method. The iterative scheme is designed particularly for voxel-based numerical simulations in which a large number of material grids issued from image processing can be envisaged. At this stage, microtomographic data available to the authors with the finest material grids are of dimensions $600 \times 500 \times 500$, from which is extracted the information on material grids located at the 300th slices. When each pixel where one material grid is 


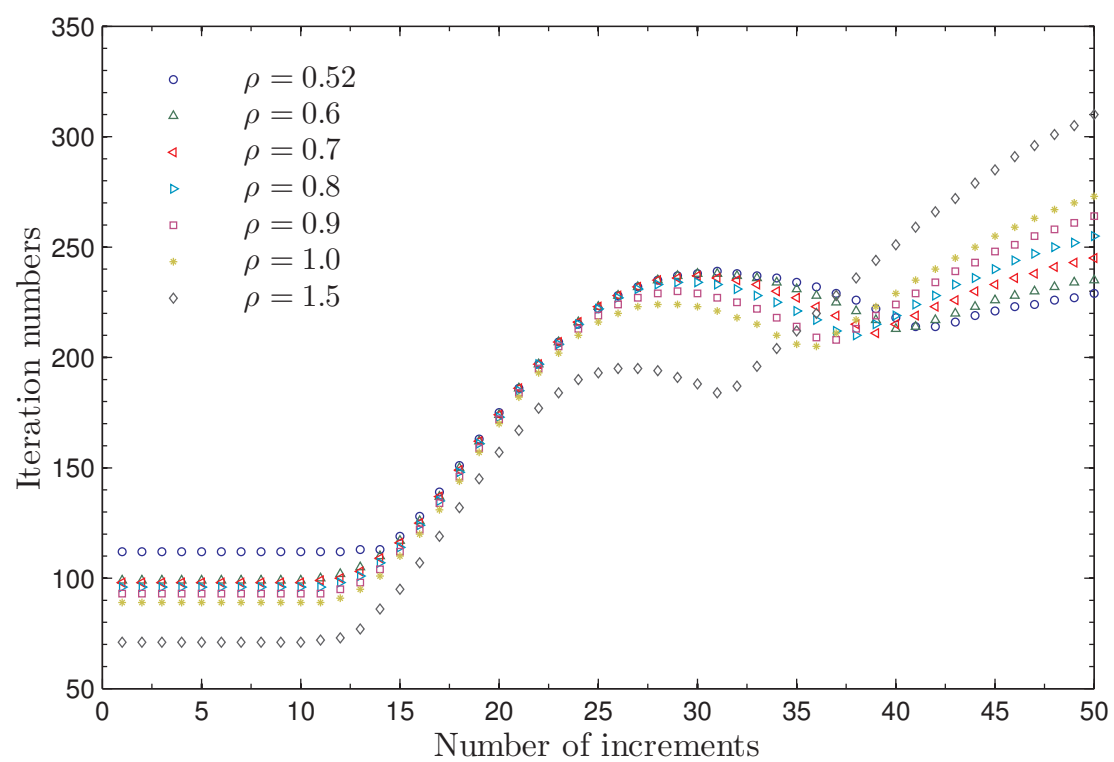

Figure 17: Numerical iteration numbers recorded during loading: comparisons between the results for different values of $\rho$

located is converted to a 4-node element for FE analyses, there is a total of $601 \times 501$ nodes and $601 \times 501 \times 2$ degrees of freedom in 2D purely elastic context. It is pointed out that the associated system stiffness matrix is symmetric but not positively definite due to Lagrange multipliers introduced to account for periodic boundary conditions. To solve such a system of equations, e.g. using MATLAB ${ }^{\circledR}$, the choice of suitable solvers is very limited. We adopt here the "symmlq" and "minres" solvers.

The proposed SLS-based iterative scheme has been parallelized. Since nonuniform damage may nucleate and propagate progressively during loading, the iteration number will vary for each strain increment. The tests are preformed on a PC station of 8 CPU cores. Each iteration costs about 6 seconds and the total time spent for one strain increment varies from 18 minutes to 20 minutes. The numerical results by the SLS-based incremental-iterative method are presented in Figure 18. As for the FE analyses with the "minres" solver, the resolution of the system equation takes about 150 minutes to converge to a relatively big tolerance $10^{-6}$, and in the nonlinear phase, one loading increment usually needs 4 iterations to converge to the prescribed global tolerance $\epsilon=10^{-3}$. In the same setting, the "symmlq" solver has shown worse efficiency than the "minres" solver. In summary, the proposed iterative scheme shows a very good efficiency: the incremental-iterative method is more than 20 times faster than the finite element method and this ratio will increase with the number of pixels (grid points).

\section{Conclusions}

An increment-enhanced iterative scheme based on the Lippmann-Schwinger and operating in the real space has been proposed in the present work for simulating damage evolution in quasi-brittle microstructures described by voxel 


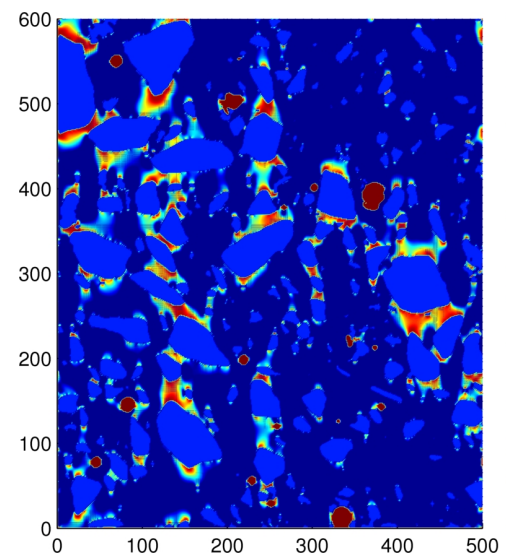

(a) 30th loading step

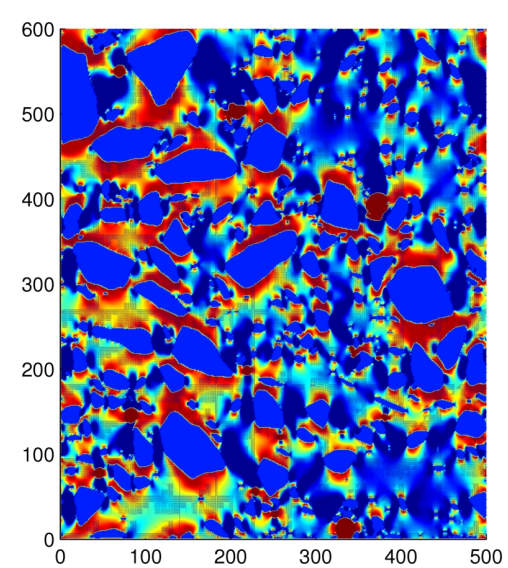

(b) 50th loading step

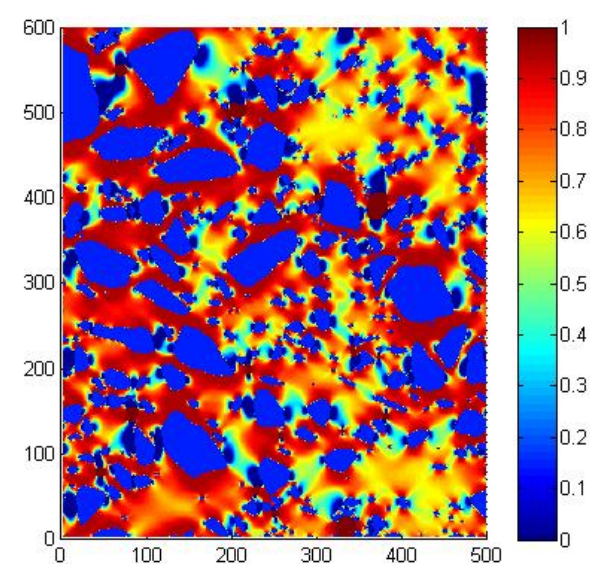

(c) 70th loading step

Figure 18: Modeling of damage evolution in a concrete with the proposed incremental-iterative method: case of $600 \times 500$ material grids

models such as those arising from microtomography images. The main scheme can be solved by general LippmannSchwinger methods like the FFT, but is developed here in the framework of the SLS method. The advantages of the technique are threefold: (a) first, no matrix needs to be assembled and decomposed; (b) second, no mesh is needed and complex geometries obtained by microtomography images can be handled; (c) a constant isotropic reference material is sufficient; (d) the scheme is shown to be very robust as compared to the FEM with Newton-Raphson algorithms in the case of softening due to damage; (e) the method is suitable for massively parallel computation software platform equipped with multi-cores and GPUs. The methodology has been validated by comparison with FEM simulations and has been shown to be very accurate and particularly efficient for large problems.

\section{Acknowledgments}

The authors would like to thank Assistant Professor Sylvain Meille and Dr. Ing. Jerome Adrien, MATEIS laboratory- UMR CNRS, University of Lyon, CNRS INSA-Lyon, France, who kindly provided the microtomography data used in the present work. The author Q.Z. Zhu thanks financial support from the National Natural Science Foundation of China (No.11202063) and from the Fundamental Research Funds for the Central Universities (No.2013B17214, 2014B06914).

[1] Salvo L, Cloetens P, Maire E, Zabler S, Blandin JJ, Buffisre JY, Josserond C (2003) X-ray micro-tomography an attractive characterisation technique in materials science. Nuclear instruments and methods in physics research section B: Beam interactions with materials and atoms 200:273-286.

[2] Sakellariou A, Arns CH (2007) Developing a virtual materials laboratory. Materials Today 10:44-51.

[3] Proudhon H, Buffiere JY, Fouvry S (2007) Three-dimensional study of a fretting crack using synchrotron X-ray micro-tomography. Eng Frac Mech 74:782-793.

[4] Young PG, Beresford-West TBH, Coward SRL, Notarberardino B, Walker B, Abdul-Aziz A (2008) An efficient approach to converting three-dimensional image data into highly accurate computational models. Philos Trans A Math Phys Eng Sci 366:3155-3173. 
[5] Stienon A, Fazekas A, Buffiere JY, Vincent A, Daguier P, Merchi F (2009) A new methodology based on X-ray micro-tomography to estimate stress concentrations around inclusions in high strength steels. Mater Sci Eng A 513 (2009) 376-383.

[6] Wei W, Sun RM, Shi YL (2010) P-wave tomographic images beneath southeastern Tibet: Investigating the mechanism of the 2008 Wenchuan earthquake. Science China Earth Sciences 53:1252-1259.

[7] Wei W, Xu JD, Zhao DP,Shi YL (2012) East Asia mantle tomography: New insight into plate subduction and intraplate volcanism. Journal of Asian Earth Sciences 60:88-103.

[8] Keyak JH, Meagher JM, Skinner HB, Motejr CD (2009) Automated three-dimensional finite element modelling of bone: a new method. J Biomed Eng 12:389-397.

[9] Maire E, Fazekas A, Salvo L, Dendievel R, Youssef S, Cloetens P, Letang JM (2003) X-ray tomography applied to the characterization of cellular materials. Related finite element modeling problems. Compos Sci Technol 63:2431-2443.

[10] Hollister SJ, Kikuchi N (1994) Homogenization theory and digital imaging: a basis for studying the mechanics and design principles of bone tissue. Biotechnol Bioeng 43:586-596.

[11] Legrain G, Cartraud P, Perreard I, Moes N (2011) An X-FEM and level set computational approach for image-based modelling: Application to homogenization. Int J Numer Methods Engng 86:915-934.

[12] Lian WD, Legrain G, Cartraud P (2013) Image-based computational homogenization and localization: comparison between X-FEM/levelset and voxel-based approaches. Comput Mech 51:279-293.

[13] Burlion N, Bernard D, Chen D (2006) X-ray microtomography: Application to microstructure analysis of a cementitious material during leaching process. Cement and Concrete Research 36:346-357.

[14] Rougelot T, Burlion N, Bernard D, Skoczylas F (2010) About microcracking due to leaching in cementitious composites: X-ray microtomography description and numerical approach. Cement and Concrete Research 40:271-283.

[15] Cebral JR, Lohner R (2001) From medical images to anatomically accurate finite element grids. Int J Numer Methods Engng 51:985-1008.

[16] Xu Y, Yagi K (2004) Automatic FEM model generation for evaluating thermal conductivity of composite with random materials arrangement. Comp Mater Sci 30:242-250.

[17] Moulinec H, Suquet P (1994) A fast numerical method for computing the linear and nonlinear mechanical properties of composites. C.R. Acad Sci II 318:1417-1423.

[18] Moulinec H, Suquet P (1998) A numerical method for computing the overall response of nonlinear composites with complex microstructure.Comp Methods Appl Mech Engng 157:69-94.

[19] Monchiet V, Bonnet G (2012) A polarization-based FFT iterative scheme for computing the effective properties of elastic composites with arbitrary contrast. Int J Numer Methods Engng 89:1419-1436.

[20] Yvonnet J (2012) A fast method for solving microstructural problems defined by digital images: a space Lippmann-Schwinger scheme.Int J Numer Methods Engng 92:178-205.

[21] Michel JC, Moulinec H, Suquet P (2001) A computational scheme for linear and nonlinear composites with arbitrary phase contrast. Int J Numer Methods Engng 52:139-160.

[22] Jiang T, Shao JF (2012) Micromechanical analysis of the nonlinear behavior of porous geomaterials based on the fast Fourier transform. Comput Geotech 46:69-74.

[23] Mishnaevsky L (2005) Automatic voxel-based generation of 3D microstructural FE models and its application to the damage analysis of composites. Mater Sci Eng A 407:11-23.

[24] Marigo JJ (1981) Formulation d'une loi d'endommagement d'un matériau élastique, C.R. Acad. Sci Paris, II 292:1309-1312.

[25] Lemaitre J (1992) A course on damage mechanics. Berlin Springer-Verlag.

[26] Ortiz M (1985) A constitutive theory for the inelastic behavior of concrete. Mech Mater 4:67-93. 